\title{
Energy-Driven Adaptive Clustering Hierarchy (EDACH) for Wireless Sensor Networks*
}

\author{
Kyung Tae Kim and Hee Yong Youn** \\ School of Information and Communications Engineering, \\ Sungkyunkwan University, Suwon, Korea \\ harisu@skku.edu, youn@ece.skku.ac.kr
}

\begin{abstract}
Wireless sensor network consists of small battery powered sensors. Therefore, energy consumption is an important issue and several schemes have been proposed to improve the lifetime of the network. In this paper we propose a new approach called energy-driven adaptive clustering hierarchy (EDACH), which evenly distributes the energy dissipation among the sensor nodes to maximize the network lifetime. This is achieved by using proxy node replacing the cluster-head of low battery power and forming more clusters in the region relatively far from the base station. Comparison with the existing schemes such as LEACH (Low-Energy Adaptive Clustering Hierarchy) and PEACH (ProxyEnabled Adaptive Clustering Hierarchy) reveals that the proposed EDACH approach significantly improves the network lifetime.
\end{abstract}

Keywords: Cluster-head, energy consumption, network lifetime, proxy node, wireless sensor networks.

\section{Introduction}

Wireless sensor networks have been evolving rapidly and now they are widely used in both the military and civilian applications such as target tracking, surveillance, and security management $[1,2]$. It is composed by hundreds or thousands of low-cost, low power, multifunctional small sensor nodes. Since a sensor is a small, lightweight, untethered, battery-powered device, it has limited energy. Therefore, energy consumption is a critical issue in wireless sensor networks [4,5]. In a sensor network a large number of sensors are deployed to carry out a given task. When sensors are deployed in the field, they establish routes and then start sensing the surroundings, computation and transmission of the data to the base station. Since sensor nodes carry limited, generally irreplaceable power source, the sensor network protocols must focus primarily on power conservation $[6,7,10]$.

The protocol called low-energy adaptive clustering hierarchy (LEACH) [3] is a cluster-based protocol proposed to solve the energy consumption problem. It equally distributes the entire energy consumption of a sensor network among the sensors, and

* This research was supported by the Ubiquitous Autonomic Computing and Network Project, 21st Century Frontier R\&D Program in Korea and the Brain Korea 21 Project in 2005.

*** Corresponding author. 
thus increases the lifetime of the network. In LEACH, however, a cluster-head can cause a failure because of energy deficiency. Sensors in a senor network are susceptible to failures due to limited battery power and will also be inactive regardless of their power condition if the cluster-head in their cluster suffers from the energy deficiency failure. If a failure occurs at the cluster-head, the network has to be re-clustered and a new schedule is transmitted to the sensors. Also, the sensor network has to stop data processing and communication in order to perform re-clustering, which requires network setup and bootstrapping. Consequently, frequent failures will result in large reclustering overhead of energy and time. Lifetime of a sensor network and its energy efficiency are thus greatly affected by the frequency of re-clustering.

PEACH (Proxy-Enabled Adaptive Clustering Hierarchy) [11] is a protocol that improves LEACH in terms of network lifetime. This is achieved by selecting a proxy node which can assume the role of the current cluster-head of weak power during one round of communication. PEACH is based on the consensus of healthy nodes for the detection and manipulation of failure in any cluster-head. It allows considerable improvement in the network lifetime by reducing the overhead of re-clustering.

In LEACH and PEACH, however, the cluster-heads are selected randomly with uniform distribution in the network. Note that energy consumption of a cluster-head will be much larger than that of cluster member nodes for the communication with the nodes inside its cluster and, more importantly, the base station. This is because energy consumption is proportional to the distance between the communicating nodes, while the base station is usually much farther than the cluster member nodes from a clusterhead. If the cluster-heads are uniformly laid out in the entire region of the sensor network, then the ones relatively far from the base station than the other cluster-heads will suffer from the energy deficiency problem more likely. Therefore, in this paper, we propose the energy-driven adaptive clustering hierarchy (EDACH) approach that puts more number of cluster-heads in the region relatively far from the base station. The number of member nodes in their clusters will then be smaller than that of other clusters. This compensates the larger energy consumption due to larger distance to the base station. It still employs the same proxy node approach as in PEACH to solve the problem of cluster-head having insufficient energy for carrying out the duty of cluster-head. If a cluster encounters a problematic cluster-head, then a proxy is selected to operate in replace of the original cluster-head. Computer simulation reveals that the proposed EDACH approach extends the network lifetime of LEACH and PEACH about $80 \%$ and $30 \%$, respectively.

The remainder of the paper is organized as follows. In Section 2 the system model including the energy model and fault model is presented along with the related works. Section 3 presents the proposed EDACH scheme. Section 4 evaluates the performance of the proposed scheme by computer simulation, and compares it with LEACH and PEACH. Finally, Section 5 concludes the paper and outlines future research directions.

\section{The System Model}

In this paper the single-hop sensor network architecture is employed for wireless sensor network as LEACH [3]. There exist three kinds of nodes in the network; sensor nodes, cluster-head nodes, and base station. The sensor nodes are used for data acquisition. The cluster-heads are for data fusion and forwarding of the aggregated infor- 
mation toward the base station. As a result, the cluster-heads consume energy at a substantially higher rate than the other nodes due to wireless communication over large distances. Consequently, the cluster-heads have shorter lifetime. Upon depletion of energy at a cluster-head, the coverage for the particular area under surveillance by that node is lost. The base station may be assumed to always have sufficient battery provisioning, or its battery may be re-provided during its course of operation. Therefore, its power consumption is not a concern in our investigation.

\subsection{The Energy Model of a Sensor}

We use the same radio model as discussed in [3, 8, 11], which is the first order radio model. In this model, a radio dissipates $E_{\text {elec }}(=50 \mathrm{~nJ} / \mathrm{bit})$ to run the transmitter or receiver circuitry and $\varepsilon_{m p}\left(=100 \mathrm{pJ} / \mathrm{bit} / \mathrm{m}^{2}\right)$ for the transmitter amplifier. The energy consumption model is described as follows. For transmission, when a node transmits $k$-bit data to another node with a distance of $d$, the energy it consumes is $E_{T x}(k, d)=$ $E_{\text {elec }} \times k+\varepsilon_{m p} \times k \times d^{2}$. For receiving, when a node receives $k$-bit data, the energy it consumes is $E_{R x}(k)=E_{\text {elec }} \times k$. Here $E_{\text {elec }}$ is the energy required for transmitting or receiving one bit data, the second term of $E_{T x}$ covers the energy loss due to channel attenuation, and $\S_{m p}$ is the amplifier coefficient. For simplicity of calculation, we assume that transmission range of each node is same on one condition that the range should cover all the neighbors in its cluster. We also assume that all data packets are same sizes. For fair comparison, we use the same constant coefficients as in LEACH.

\subsection{The Fault Model}

Data transmission failure occurs when a cluster-head cannot transmit data due to energy deficiency. Failure at a cluster-head affects the system status and causes a remedial operation such as re-clustering or boot-strapping that results in increased network down time and reduced network lifetime, etc. We assume that the data in the communication are error-free and the semantic-related generic faults during data transmission can be detected and removed by the application specific operation. Data transmission failure at a cluster-head can be caused by hardware failure or energy deficiency.

Failure at a cluster-head prevents it from transmitting data to the sensors as well as relaying the data to the base station. The data sent by the sensor nodes will also be lost. Once a cluster-head fails, it can no longer serve as a liaison between the sensor nodes and the base station. The failure at the cluster-head is tried to be avoided using the proxy nodes and non-uniform distribution of the cluster-heads as explained later. In our fault model we consider only the failure at the cluster-heads.

With LEACH, the cluster-heads are stochastically selected. In order to select the cluster-heads, each node generates a random number between 0 and 1 . If the number is smaller than the threshold, $T$, the node becomes a cluster-head for the current round. The threshold is calculated as follows.

$$
\mathrm{T}=\frac{\mathrm{P}}{1-\mathrm{P} \times\left(\operatorname{r\operatorname {mod}} \frac{1}{\mathrm{P}}\right)}
$$


Here $P$ is the portion of the nodes becoming the cluster-heads and $r$ is the number of current round. If a node once has been a cluster-head in the last $1 / P$ rounds, it cannot be a cluster-head again. This algorithm thus ensures that every node becomes a cluster-head exactly once within $1 / P$ rounds.

Refer to Figure 1(a) of an example of clustering with LEACH and PEACH, where five cluster-heads (the black dots) are selected randomly among 100 nodes with uniform distribution. Here the base station is assumed to be located outside the region at the upper-right corner direction. Since the cluster-heads were selected randomly with uniform distribution, the ones far from the base station will consume their energy more quickly than the others as mentioned earlier. Therefore, we propose to put more cluster-heads in the area of the nodes far from the base station. This can be easily implemented by setting the $P$ value of Equation (1) differently. For example, as shown in Figure 1(b), the entire range is partitioned into three segments, and the $P$ value of the nodes in the segment close or far from the base station is decremented or incremented, respectively. This approach is expected to evenly distribute energy consumption among the nodes, and thus outperform the existing approaches having uniform distribution of cluster-heads. The proposed scheme is presented next.

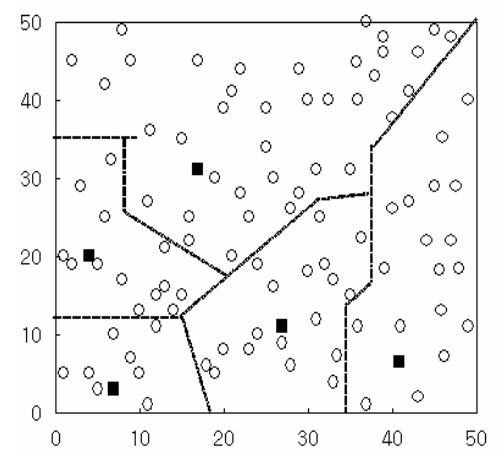

(a) Clustering with LEACH and PEACH

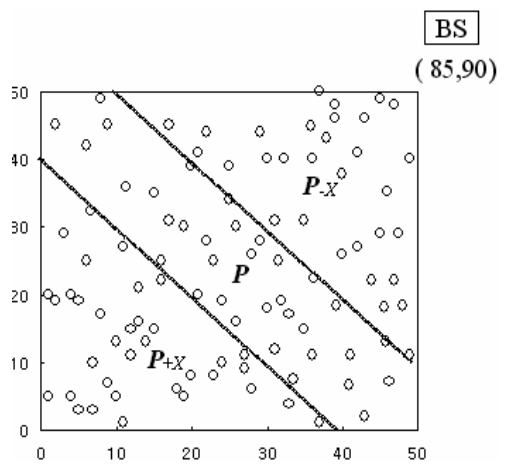

(b) Partition of the nodes

Fig. 1. An example of clustering and node partitioning

\section{The Proposed Scheme}

We call the proposed protocol as energy-driven adaptive clustering hierarchy $(\mathrm{EDACH})$, which is an enhanced version of the LEACH and PEACH scheme. One round of its operation consists of the following two phases: i) set-up phase, ii) selforganized data collection and transmission phase. The proposed scheme is to solve the possible problem of the cluster-head in the LEACH approach having insufficient energy for carrying out the duty of cluster-head by using proxy nodes like the PEACH approach. It, however, further improves the performance of PEACH by forming more clusters in the region relatively far from the base station.

Each round of communication in the proposed scheme begins with the set-up phase where the clusters are organized, followed by the self-organized data collection and 
transmission phase where data transfer to the base station occurs. The second phase also includes the proxy node selection process and Indicator Control Message (ICM) advertisement process. We next introduce the EDACH protocol in detail.

\subsection{The Set-Up Phase}

In LEACH and PEACH, a fixed portion of the sensors stochastically decides themselves as cluster-heads as described in $[3,11]$. The problem of cluster-head population in a wireless sensor network was analyzed in [3]. They showed that $5 \%$ of the nodes in the network operating as cluster-heads can achieve good performance in a homogeneous network with various parameter settings. Recall that we propose to regulate the number of cluster-heads according to the proximity to the base station. For this, we partition the entire area of the sensor network into three segments as near, medium, and far segment as shown in Figure 1(b). Of course, the shape of partition will be different according to the position of the base station, and the number of segments could also be varied.

In the set-up phase, each node calculates the threshold using Equation (1), but the values of $P$ for the nodes belonging to different segments differ from each other. That is, the $P$ value for the nodes in the near, medium, and far segment is $(1-x) P, P$, and $(1+x) P$, respectively, where $0<x<1$. With this arrangement the nodes relatively close to the base station will have smaller threshold value and thus smaller chance to become a cluster-head. As a result, the number of cluster-heads in that segment will be relatively small. Computer simulation reveals that the proposed approach increases the network lifetime compared to PEACH. Figure 2 shows the result of clustering with EDACH for the same distribution of nodes as in Figure 1.

When a node is selected as a cluster-head, it generates a cluster-head token. Then, the selected cluster-head advertises its token by CSMA/CA MAC protocol to all its neighbors. After the remaining nodes receive the advertisements, they compare the strengths of the received signals. It keeps only the token with the strongest signal in every comparison, and randomly chooses a one if a tie occurs.

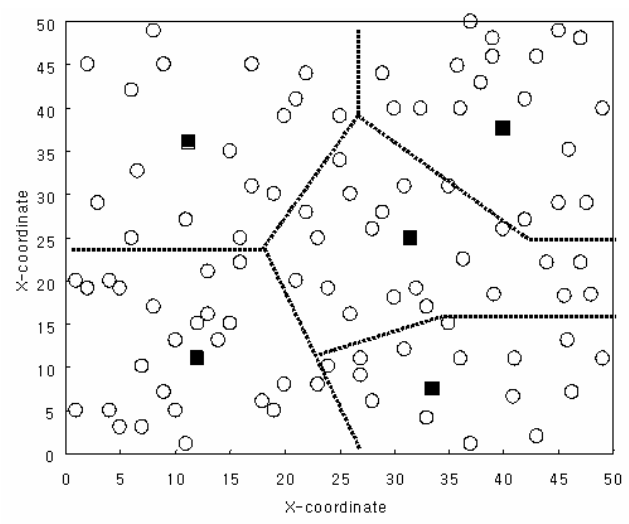

Fig. 2. An example of clustering with $\mathrm{EDACH}$ after the set-up phase 
After the advertisement, every cluster member node recognizes the source of the token as its cluster-head and broadcasts the topology reply packet by CSMA/CA MAC protocol back to the cluster-head. In the reply packet the node's position (NP) and remaining energy (RE) level are included. When the cluster-heads receive the reply packets, they set up a schedule for the nodes in their cluster. Based on the number of nodes in the cluster, the cluster-head creates a TDMA schedule indicating when each node in the cluster can transmit.

\subsection{The Self-organized Data Collection and Transmission Phase}

After the set-up phase, the self-organized data collection and transmission phase starts. Every sensor node collects the data and then sends a packet to the cluster-head in its scheduled transmission time. The radio of a node is turned off when it is outside its scheduled time slot in order to save the energy. Each cluster-head keeps its receiver on to collect data from the nodes in its cluster and continuously updates the table listing the energy of the nodes based on the received packets. When the data from all the nodes have been received, the cluster-head executes the function for data fusion to aggregate the received data into one packet. After the data fusion, the cluster-head sends it to the base station. As a cluster-head needs to receive many packets and consume large power for long range transmission, its energy is used up more quickly than other nodes in the cluster. Therefore, a cluster-head can cause a failure because of energy deficiency. If a failure occurs at a cluster-head, the network has to be re-clustered and a new schedule needs to be transmitted to the sensors. This will significantly reduce the network lifetime. In order to solve this problem, a proxy node is selected if the battery power of the cluster-head becomes smaller than the threshold explained next.

Calculation of the Threshold Value. The threshold value, $\boldsymbol{E}_{T H}$, plays a very important role in the data transmission phase since it is used as a measure for deciding if the current cluster-head has become obsolete. It is calculated when a cluster-head is selected. If the energy of the cluster-head drops below the threshold, the proxy node selection process begins. We assume that all sensors are identical and produce data at the same rate. The following equations are used to compute the threshold value.

$$
\begin{gathered}
k_{j}=M_{b i t} \sum_{i=1}^{\left[\frac{n}{k}\right]_{1}} k_{i j} \\
E_{C H(j)}=E_{\text {elec }} \times k_{j}+\varepsilon_{\text {amp }} \times k_{j} \times d_{C H(j)}^{2} \\
E_{T H}=\frac{1}{k} \sum_{j=1}^{k} E_{C H(j)}
\end{gathered}
$$

Here $k_{j}$ is the length of the aggregated message in the cluster-head and $d_{C H}$ is the distance between the cluster-head and base station. Since $E_{T H}$ changes with time, it is recalculated in every self-organized data collection and transmission phase.

When the energy level of the cluster-head falls below the threshold, a proxy node is selected using the RE and NP value of the reply packets received in the set-up phase (Refer to [11]). After a node is selected as a proxy node, the cluster-head 
broadcasts an indicator control message (ICM) containing the address of the proxy node and a new TDMA schedule for the member nodes. The member nodes receiving the ICM send a confirmation message to the proxy node. When the proxy node receives the confirmation message, it keeps the node IDs of the member nodes. After the message exchanges are over, the member nodes resume data transmission. The ICM advertisement process is described in Figure 3.

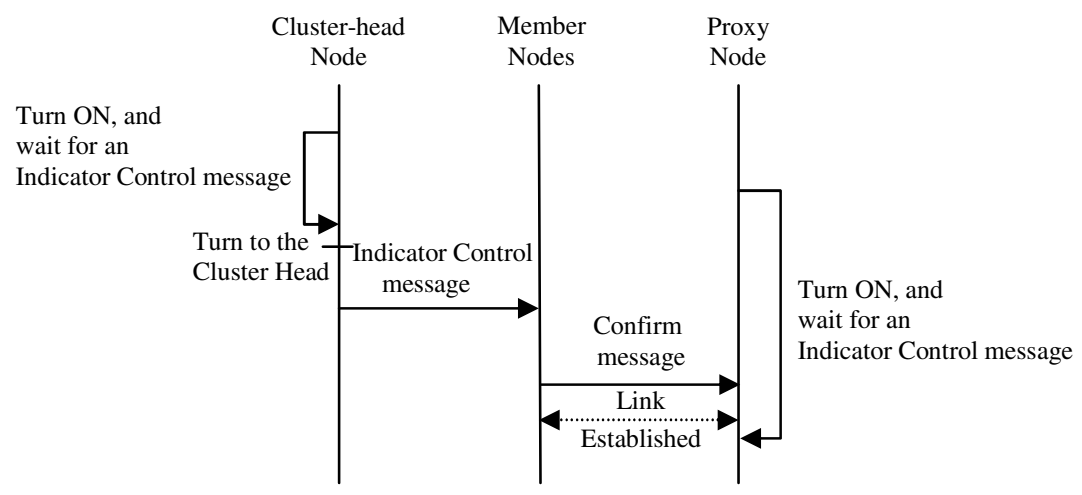

Fig. 3. The sequence of operations in the ICM advertisement process

\section{Performance Evaluation}

We evaluate the effectiveness of the proposed EDACH scheme along with LEACH and PEACH through computer simulation. The probability for a node to be selected as a cluster-head is set to $5 \%$. For the simulation we consider a sensor network of 100 sensor nodes randomly arranged in a $50 \times 50$ region. A base station is located at $(85$, 90). We use two models of initial residual energy of sensor nodes; uniform at $0.5 \mathrm{~J}$ and random between $0.25 \mathrm{~J}$ and $0.5 \mathrm{~J}$. We set $E_{\text {elec }}$ to $50(\mathrm{~nJ} / \mathrm{bit})$ and $\varepsilon_{\text {amp }}$ to $100\left(\mathrm{pJ} / \mathrm{bit} / \mathrm{m}^{2}\right)$ in the energy model of a sensor. The size of sensor data is $2000 \mathrm{bits}$, and the advertisement message is 64-bit long. The value of $x$ in the $(1-x) P$ and $(1+x) P$ formula mentioned earlier is set to 0.2 . In the simulation the result of 100,000 runs are averaged.

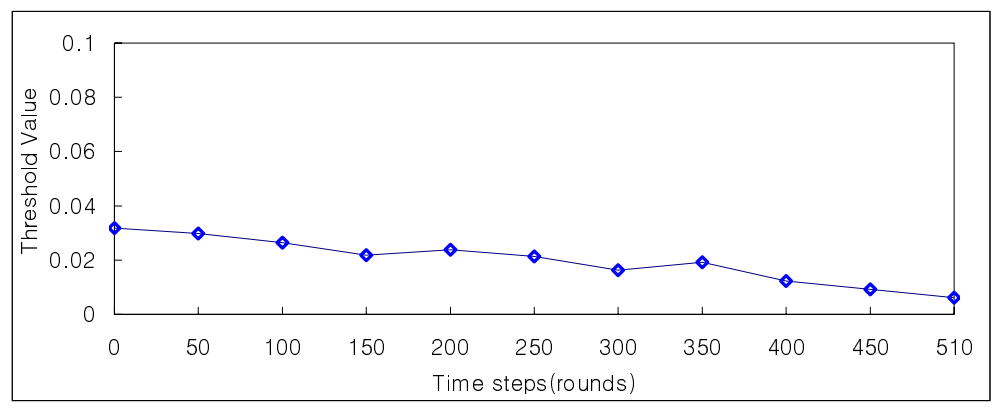

Fig. 4. The threshold values obtained as time moves 
Figure 4 shows the threshold values obtained as time moves. When $E_{T H}$ is large, a proxy node is more likely selected to take over the role of the cluster-head and thus the cluster-head can reserve energy for later use. In this sense proxy node can help the cluster-head have a longer life.

Table 1 lists the lifetime of the sensor network in terms of the round a node begins to die and the round the last node dies for the three schemes compared. Notice that the proposed EDACH protocol is consistently better than the others. Especially, the proposed EDACH outperforms LEACH and PEACH more significantly when the initial energy is relatively high. We ran the simulator with different energy thresholds and obtained similar results.

Table 1. The network lifetimes with different initial energies of the sensors

\begin{tabular}{|c|c|c|c|}
\hline $\begin{array}{c}\text { Energy } \\
(\mathrm{J} / \text { node })\end{array}$ & Protocol & The round a node begins to die & The round a node begins to dies \\
\hline \multirow{3}{*}{0.25} & Direct & 52 & 112 \\
\cline { 2 - 4 } & LEACH & 228 & 353 \\
\cline { 2 - 4 } & PEACH & 294 & 492 \\
\cline { 2 - 4 } & EDACH & 347 & 529 \\
\hline \multirow{3}{*}{0.5} & Direct & 104 & 211 \\
\cline { 2 - 4 } & LEACH & 498 & 756 \\
\cline { 2 - 4 } & PEACH & 795 & 1029 \\
\cline { 2 - 4 } & EDACH & 953 & 1358 \\
\hline
\end{tabular}

The improvement offered by the proposed EDACH protocol over LEACH and PEACH can be clearly seen in Figure 5, which shows the number of sensors alive as the round proceeds with $0.25 \mathrm{~J} /$ node initially. A sensor node with insufficient residual energy can occasionally become a cluster-head even though there is a sensor node with more battery power nearby. It then exhausts the energy, stops operating, and disrupts gathering data in its cluster. Also, data transmission to the base station is not possible. On the other hand, in the proposed protocol, the approach of proxy node and distribution of cluster-heads considering the distance to the base station allows significantly increased network lifetime.

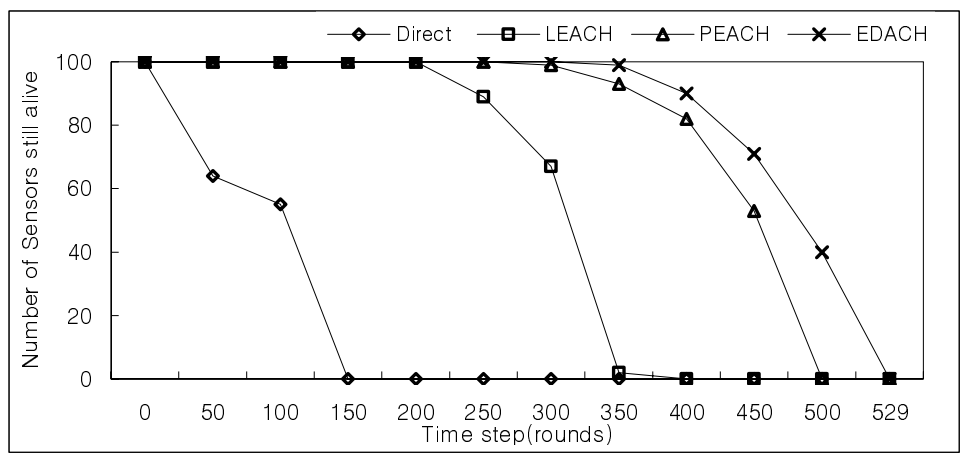

Fig. 5. Comparison of the number of live sensors as the round proceeds 
Another important aspect of the proposed protocol is illustrated in Figure 6, which shows the locations of live (circle) and dead (dot) sensor nodes with PEACH and EDACH, respectively, after 480 rounds. Observe that, in addition to a lot more live nodes than PEACH, EDACH displays well dispersed live nodes. This will be an important aspect that the proposed EDACH protocol can avoid dead spot and extend the lifetime of the network. This was achieved by employing the proxy node approach for weak cluster-heads and non-uniform distribution of them.

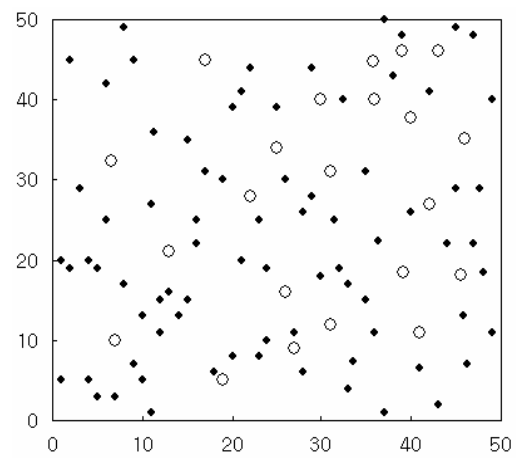

(a) $\mathrm{PEACH}$

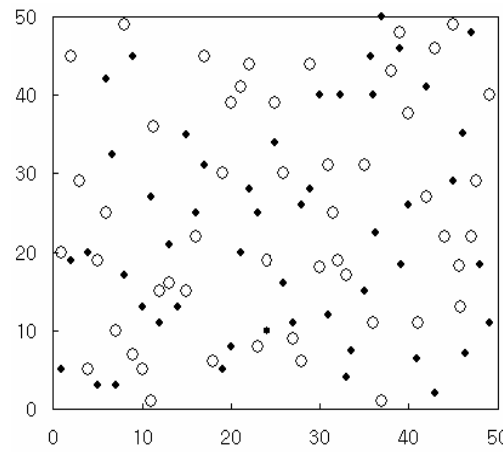

(b) $\mathrm{EDACH}$

Fig. 6. The distribution of live (circle) and dead (dot) nodes after 480 rounds

\section{Conclusion and Future Work}

In this paper we have proposed a protocol called EDACH that solves the problem of cluster-head having low energy using the proxy node approach which assumes the role of the cluster-head in the wireless sensor network. The proposed protocol further enhances the network lifetime by distributing the cluster-heads according to the distance to the base station. Computer simulation results showed that the proposed approach allows much longer lifetime of wireless sensor network than the existing schemes. The proposed approach will be more important when the wireless sensor network is deployed in large area and the base station is far from the network.

The future work will focus on the comparison of the EDACH approach with other approaches such as simulated annealing and taboos search. In the proposed EDACH approach several factors have been decided heuristically such as the number of partitioned segments, the threshold energy value used for deciding whether a proxy node is required or not, etc. A formal methodology will be developed in order to determine such factors in a more systematic way and also to allow optimal values for the given conditions. In addition, the proposed approach will be extended to multi-level cluster hierarchy. 


\section{References}

[1] L. Zhong, R. Shah, C. Guo, J. Rabaey: An ultra low power and distributed access protocol for broadband wireless sensor networks: IEEE Broadband Wireless Summit, Las Vegas, May 2001.

[2] I.F. Akyildiz, W. Su, Y. Sankarasubramaniam and E. Cayirci.: Wireless sensor networks: a survey Computer Networks: 38 (4) (2002) pp. 393-422

[3] W.R.Heinzelman, A.Chandrakasan, and H. Balakrishnan.: Energy-Efficient Communication Protocol for Wireless Micro-sensor Networks: In Proceedings of the Hawaii International Conference on System Science, Maui, Hawaii,2000.

[4] A. Sinha, A. Chandrakasan.: Dynamic power management in wireless sensor networks: IEEE Design \& Test of Computers, March-April 2001, S. 62-74.

[5] L. Zhong, R. Shah, C. Guo, J. Rabaey.: An ultra low power and distributed access protocol for broadband wireless sensor networks: IEEE Broadband Wireless Summit, May 2001.

[6] G. Gupta and M. Younis.: Fault-Tolerant Clustering of Wireless Sensor Networks: IEEE Wireless Communications and Networking, Vol. 3 pp. 1577-1584, Mar 2003.

[7] K. Sohrabi, J. Gao, V. Ailawadhi, G.J. Pottie.: Protocols for selforganization of a wireless sensor Network: IEEE Personal Communications, October 2000, pp. 16-27.

[8] W. Heinzelman, A. Chandrakasan, H. Balakrishnan.: An applicationspecific protocol architecture for wireless microsensor networks: in press: IEEE Trans. on Wireless Networking.

[9] G. Gupta and M. Younis.: Load-balaned clustering of wireless sensor networks: IEEE ICC, 1848-1852, May 2003.

[10] MJ Handy, M. Haase, D. Timmermann.: Low-Energy Adaptive Clustering Hierarchy with Deterministic Cluster-Head Selection: August 2002.

[11] K.T. Kim and H.Y. Youn.: PEACH: Proxy-Enable Adaptive Clustering Hierarchy for Wireless Sensor network: Proceeding of The 2005 International Conference On Wireless Network, June 2005, pp. 52-57. 